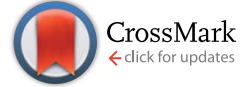

Cite this: RSC Adv., 2015, 5, 48399

Received 1st May 2015

Accepted 21st May 2015

DOI: $10.1039 / c 5 r a 08044 d$

www.rsc.org/advances

\section{Continuous and low error-rate passive synchronization of pre-formed droplets $\uparrow$}

\author{
O. J. Dressler, ${ }^{a}$ T. Yang, ${ }^{\text {a S. }}$-I. Chang, ${ }^{\text {b J J. Choo, }},{ }^{\text {C R. C. R. Wootton }}{ }^{\mathrm{a}}$ and A. J. deMello*a \\ A microfluidic droplet-handling architecture for the synchronization of asynchronous, mis-matched, pre- \\ formed droplet streams is demonstrated. This architecture is shown to be robust to variations in droplet \\ input frequencies, whilst still producing highly reliable synchronisation. The operational phase space with \\ regards to droplet size disparity is explored and the long-term operational stability of the system \\ confirmed. Specifically, the microfluidic platform to synchronies droplet streams at a rate of $33 \mathrm{~Hz}$ over \\ extended periods of time and with an error rate less than $0.2 \%$.
}

\section{Introduction}

In recent years droplet-based microfluidics has matured into a versatile platform for high-throughput biological and chemical experimentation., ${ }^{\mathbf{1} 2}$ The miniaturization and planarization of biological and chemical assays implied by microfluidic experimentation allows for significantly increased experimental throughput, decreased instrumental footprints, decreased reagent consumption and exceptional control over experimental conditions. ${ }^{3-5}$ It is also recognized that microfluidic systems offer an ideal environment for the controlled formation of emulsions or segmented flows. ${ }^{6}$ Compared with bulk emulsification techniques, microfluidic strategies can produce droplet populations with negligible polydispersity across a wide range of droplet diameters. ${ }^{7}$

Enclosing droplets (that contain for example assay reagents) in an immiscible carrier fluid allows for precise control of reactions in both time and space. Each droplet may be considered to be an isolated reaction container ${ }^{8}$ separated from the other droplets by the carrier fluid, effectively eliminating cross-contamination between samples. ${ }^{9}$ Typical microfluidic architectures produce droplets with volumes down to the low picoliter range and at $\mathrm{kHz}$ frequencies. ${ }^{\mathbf{1 0 1 1}}$ Several chip-based architectures for the formation of highly monodisperse droplets have been demonstrated, including T-junctions (where droplet formation is induced by a pressure drop), ${ }^{\mathbf{8} 12} \mathrm{~V}$-junctions (that support a wider combination of droplet sizes, spacings and generation frequencies) ${ }^{13}$ and flow-focusing junctions (where droplet formation is induced by capillary number

${ }^{a}$ Institute for Chemical and Bioengineering, Department of Chemistry and Applied Biosciences, ETH Zürich, Vladimir Prelog Weg 1, 8093 Zürich, Switzerland. E-mail: andrew@ethz.ch

${ }^{b}$ Department of Biochemistry, Chungbuk National University, Cheongju, South Korea ${ }^{c}$ Department of BionanoTechnology, Hanyang University, Ansan 426-791, South Korea † Electronic supplementary information (ESI) available: See DOI: 10.1039/c5ra08044d related instabilities). ${ }^{\mathbf{1 4}}$ These strategies exploit shear forces present at the interface between two immiscible phases to split a continuous fluid flow into separate droplets without requiring any external forces such as ultrasound or electrical fields. ${ }^{\mathbf{1 5}}$ Typically droplet size, shape and formation rate are dependent on a multitude of factors such as input flow rates, interfacial surface tension and viscosities of the two immiscible phases used. Other important factors include the channel geometry and channel surface properties. It is also recognized that surfactants are almost always used to stabilize droplets subsequent to formation. ${ }^{5}$

In recent years there has been a trend towards the establishment of complex biological assays using droplet-based microfluidic systems. ${ }^{2,7,16,17}$ Such assays are based on the sequential performance of defined unit operations. ${ }^{7}$ Droplets may be split, ${ }^{18,19}$ trapped, ${ }^{20}$ diluted $^{21}$ and incubated over extended periods of time..$^{22-26}$ Sorting of droplets based on content ${ }^{15,27-29}$ or morphology ${ }^{30}$ can be realized using a range of strategies, and droplet merging, an essential step in the implementation of almost any assay, can be achieved both passively and actively. ${ }^{31-34}$ It is also important to note that effective methods for pairing droplets in an A-B pattern can prove extremely useful when probing inter-droplet transfer, ${ }^{35}$ contacting droplets using shift registers ${ }^{36}$ or initiating cell fusion. ${ }^{37}$

These functions have been used for a range of applications, including the assessment of ultra-fast reactions, ${ }^{38}$ multi droplet traps to study osmotic transport, ${ }^{39}$ on-demand formation of single droplets, ${ }^{\mathbf{4 0}}$ spatial encoding of individual droplets, ${ }^{\mathbf{4 1}}$ creation of a droplet populations with combinatorial chemical probes $^{40}$ as well as self-assembly of droplets into complex threedimensional structures. ${ }^{41}$ In all these studies, the combination of individual toolbox functions to create the assay requires precise control over the spatial and temporal ordering of individual droplets to ensure experimental reproducibility. Droplet synchronization can be achieved using active architectures 
where each droplet is addressed and manipulated on an individual basis. For example, dosing reagents into droplets can be achieved using pico-injectors ${ }^{42}$ or on-chip mechanical valving. ${ }^{43}$ Active dosing can be performed at high speeds but requires complex control architecture and in-line process monitoring. ${ }^{\mathbf{4 2 , 4 3}}$ Conversely, passive droplet synchronization provides the experimenter with control over droplet ordering whilst requiring little added complexity. The simplest mode of passive synchronization operation arranges two types of droplets into an alternating sequence prior to a droplet merging architecture..$^{21,43-46}$ In such a system it is crucial to minimize synchronization errors so as to ensure a constant composition of the merged droplet stream.

Streams of alternating (A-B) droplets have previously been produced using coupled droplet formation schemes. For example, combined T-junctions can be used to form the droplets in parallel. ${ }^{4-50}$ Interestingly, Frenz and co-workers utilized a similar approach to form A-B droplet streams with an error rates as low as 1 in a million droplets. ${ }^{51}$ A related approach by Hong and co-workers coupled two droplet forming junctions using a passive pressure oscillator to achieve a similar level of synchronization. ${ }^{45}$ Finally, step emulsification can also be used to form streams of alternating droplets. ${ }^{52}$ In step emulsification the dispersed phase is injected through a small channel into a higher channel filled with the continuous phase. When two step-emulsification junctions are placed in close proximity and are fluidically close-coupled the interplay leads to the formation of A-B droplet streams. ${ }^{53}$

Although a number of approaches for forming A-B alternate droplets have been shown, the operation of more than two droplet forming junctions in parallel usually leads to large disparities in both droplet size and formation frequency. ${ }^{\mathbf{1 2 , 5 4}}$ Accordingly, previous approaches based on controlling droplet formation have been limited in the maximal distance between the point of droplet formation and the point of merging so as to allow close coupling, and thus do not allow subsequent merging events to be performed in a controllable manner.

Given that droplet merging is only one of a series of operations that may be performed within an assay and that it may not always be the first operation, the inability to couple pre-formed droplets in a high-throughput manner is a significant drawback. Consequently, an optimal droplet synchronization architecture should process pre-formed droplets, such that droplet formation is handled independently from all downstream operations. Several studies have shown synchronization of preformed droplets or bubbles. For example Prakash and Gershenfeld demonstrated the removal of timing errors between two streams of gas bubbles using a fluidic ladder. ${ }^{55}$ In this case, a bubble traversing the ladder is slowed down by diversion of the oil flow through the alternate path. When two bubbles are present simultaneously, there is a net flow from the channel containing the leading bubble to the one containing the lagging bubble, creating a velocity gradient that disappears as the bubbles synchronize. This concept has been adapted for use with droplets but accurate synchronization requires precise control over both droplet size and formation rate. ${ }^{47}$ If droplets are formed using a relatively low oil fraction, spontaneous pattern formation can be observed. ${ }^{56}$ For example A-B patterns have been formed using a single T-junction ${ }^{6}$ or multiple $\mathrm{T}$ junctions in parallel. ${ }^{12}$ Step emulsification of two dropletforming phases into a single channel can also lead to a densely-packed "zig-zag" arrangement. ${ }^{52}$ Oil removal from a stream of pre-formed droplets leading to a more densely packed droplet stream can be achieved using a simple pillar array. ${ }^{31}$ Surenjav and co-authors have shown that different patterns can be formed and manipulated by varying the channel geometry and droplet size. ${ }^{57}$ Furthermore, reorganization of droplets in an ordered pattern flowing around a bend has been characterized. $^{7}$

In practice the frequency of droplet formation fluctuates over time due to pressure and flow rate instabilities. These frequency fluctuations increase over longer time scales, primarily due to droplets "catching up" with each other, which leads to groups of droplets travelling together rather than as a regular, dispersed stream. It is therefore essential that a low-error droplet synchronizer be able to compensate for variations in droplet frequency and size. Without such a buffering operation synchronization efficiency will be dependent on the formation frequency. Using the fact that smaller droplets travel at higher speeds than larger ones, Mazutis and co-workers merged two streams of heterogeneous droplets using a zig-zag channel and altered surfactant conditions. ${ }^{23}$ This approach could be used to synchronize droplets even if an excess of smaller droplets is present. Nevertheless, such an approach is limited to droplets of different size and where only the smaller ones can be present in excess.

To address the aforementioned inadequacies we present herein a structure for the continuous and passive synchronization of pre-formed droplets with very low error rates. Our approach involves densely packing two types of droplets and coinjecting them into a channel to form an ordered A-B alternate pattern. This pattern is then rearranged into a single stream of alternating droplets. The high error-tolerance results as a consequence of a buffer structure that allows the removal of excess droplets during the packing process.

\section{Experimental}

Fig. 1a shows a schematic of the microfluidic device used for droplet synchronization. The primary components include two flow-focusing droplet generators, the synchronization architecture (highlighted in Fig. 1b) consisting of a packing chamber, a co-injection channel and a constriction that moves synchronized droplets into an alternating order. Significantly, we also include a route for droplets to exit the packing chamber when the chamber becomes full (overflow mode). Such an overflow capability reduces the impact of any errors in the upstream droplet formation process and ensures stable droplet coinjection into the common channel. It should also be noted that the two "Out 2" channels were only recombined for fabrication purposes and we have successfully operated devices with distinct outlets, allowing for independent control of backpressure within these channels. Fig. 1c shows an image of the synchronization architecture in operation. 
(a)

(b)

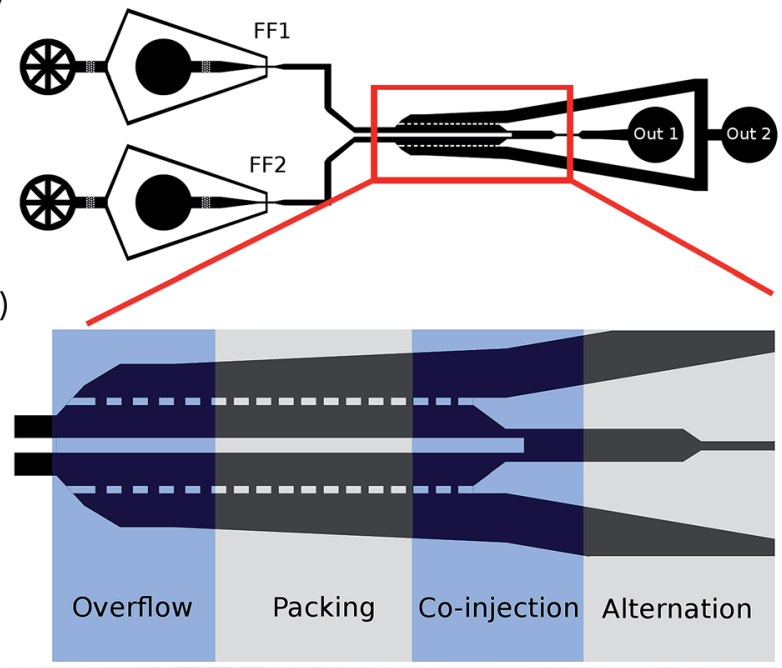

(c)

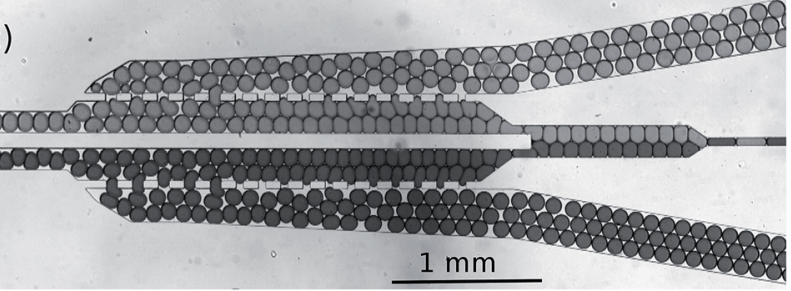

Fig. 1 Device architecture (scale bar $=1000 \mu \mathrm{m}$ ). (a) The complete device includes two independent flow-focusing junctions for droplet formation and a droplet synchronization chamber. (b) Enlarged micrograph of the droplet synchronization chamber. Droplets can exit readily through the $75 \mu \mathrm{m}$ gaps between the pillars in the overflow section. Droplets are further compacted in the packing section, through drainage of the continuous oil phase and retention of droplets. Droplets are then alternately injected into a common channel that narrows to push the droplets in an alternating order. (c) Image of the microfluidic device in operation.

Fig. 2 shows a series of images following a set of four droplets as they pass through the synchronization architecture. After removal of most of the spacer oil (upstream of the field of view), each input channel is constricted so that droplets are forced move in single file. This results in the droplets fully occupying the main synchronization channel in a zig-zag configuration. If the width of this channel is further reduced, the two rows of droplets collapse into a single file of alternating droplets. To investigate the performance of the synchronization architecture, we assessed the synchronization efficiency using a variety of droplet sizes and total flow rates. Additionally, the long-term performance of the architecture was assessed, as well as the ability to split the synchronized stream into the two original separate droplet streams.

Microfluidic devices were fabricated via conventional soft lithographic methods in polydimethylsiloxane (PDMS). ${ }^{6}$ The PDMS base and curing agent (Sylgard 184; Dow Corning, Midland, USA) were mixed at a ratio of $10: 1 \mathrm{wt} / \mathrm{wt}$, degassed and decanted onto the master. The entire structure was cured in the oven at $70^{\circ} \mathrm{C}$ overnight and then peeled off the master. After punching inlet and outlet vias through the structured PDMS layer, the PDMS was bonded to another flat PDMS substrate

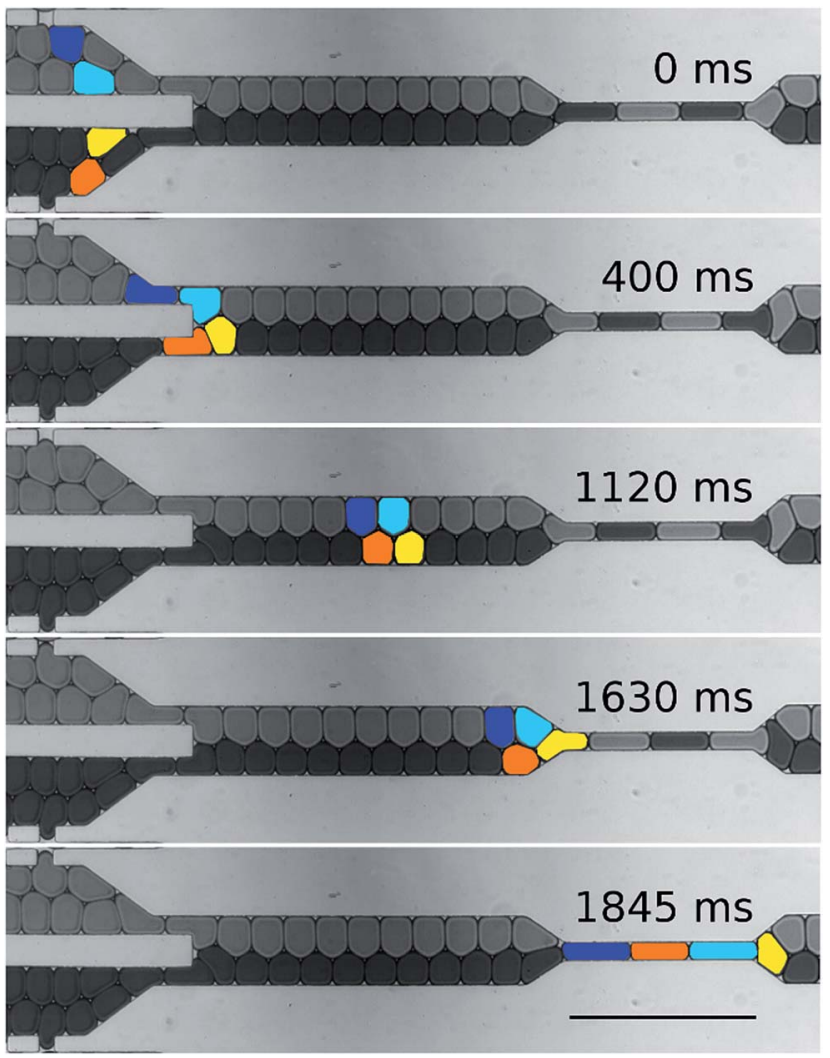

Fig. 2 A series of images extracted from a high-speed video (Movie S1 $\dagger$ ) showing a set of droplets moving through the synchronization architecture (scale bar $-600 \mu \mathrm{m}$ ). Droplets are passively ordered into an A-B alternating pattern when they are injected into the common channel (400 ms into the video). Once this channel is constricted so as to only allow a single file of droplets ( $1600 \mathrm{~ms}$ into the video) the two rows of droplets are impelled into an A-B sequential order.

using an oxygen plasma and then laid on a hot plate at $95^{\circ} \mathrm{C}$ for at least 2 hours to complete the bonding process. The height of all channels across the device was measured to be $50 \mu \mathrm{m}$.

A mixture of Fluorinert ${ }^{\mathrm{TM}} \mathrm{FC}-40$ (3M, St. Paul, MN, USA) and 4\% EA-surfactant (RainDance Technologies, Billerica, USA) was used as the continuous phase. Ink (Waterman, Paris, France) diluted with deionised water was used to form the dispersed phase. neMESYS low pressure dosing modules (Cetoni GmbH, Korbussen, Germany) were used to pump fluids at a rate of $1 \mu \mathrm{l}$ $\min ^{-1}$ using $1 \mathrm{ml}$ gastight syringes (Hamilton Bonaduz AG, Bonaduz, Switzerland).

A MotionPro Y5 Compact Digital Camera (IDT, Hitchin, United Kingdom) was used to image the passage of droplets through the constriction at 200 frames per second. A time series of the average pixel intensity in a defined region of interest was extracted from the image data using ImageJ (National Institutes of Health, Bethesda, USA) and further processed using customwritten Python (Python Software Foundation, Beaverton, USA) scripts. Specifically, a threshold to differentiate between different coloured droplets was selected manually and packet lengths calculated from the time between transits over the threshold value. Using a histogram of packet lengths, the number of droplets per packet was then mapped to the packet 
length. Synchronization performance (defined as the percentage of error-free alternating droplets) was determined by iterating over all packets in a series and counting the number of packets where both the packet itself and the next packet consisted of a single droplet.

\section{Results and discussion}

Fig. 3a reports the droplet synchronization efficiency of the device for a range of droplet sizes, with Fig. $3 \mathrm{~b}$ illustrating the variability in synchronization performance across all data points $(N=3$, with an average of 2000 synchronized droplets per experiment). Inspection of Fig. 3a indicates that the current device performs optimally within a defined region of phase space, synchronizing two equally-sized droplet populations with a diameter of $126 \mu \mathrm{m}$. Given the synchronization channel has a width of $220 \mu \mathrm{m}$, optimal droplet diameters represent $58 \%$ of this width, which allows two rows of droplets to fully occupy the main channel in a side-by-side arrangement. Variability data (Fig. 3b) further confirm that synchronization efficiencies could be obtained repeatedly and reliably. Additionally it could be seen that synchronization performance as well as reliability drops dramatically, when both droplets have a diameter smaller than $107 \mu \mathrm{m}$.

The key concept behind the presented architecture is the coinjection of densely packed droplets into a common channel. Since droplets are deformed prior entering the common channel, a significant increase in hydrodynamic resistance is generated through Laplace pressure build-up. ${ }^{58}$ This pressure increase leads to a delay of the following droplet, favouring passage of droplets entering from the other channel. This results in droplets being injected into the common channel in an alternating fashion. After injection, these droplets rearrange into an A-B alternate pattern through minimization of surface area. Once a stable A-B alternate conformation is achieved droplets collapse into an alternating order through a reduction in the channel width.

Given a common need to pack droplets in an A-B configuration it is apparent that the performance of the synchronization architecture depends on both device geometry and droplet size. It is to be expected that the optimal diameter of the droplets synchronized should be marginally larger than half the width of the common channel, due to the need to densely pack two rows of droplets into this channel. Fig. 3 confirms that optimal synchronization could indeed be achieved using equally sized droplets with a diameter of $126 \mu \mathrm{m}$, which is approximately half the width of the common channel $(220 \mu \mathrm{m})$. Additionally, reliable synchronization could also be achieved with droplets of different sizes (with up to a $15 \%$ difference from the optimal diameter) as long as the sum of both droplet diameters is slightly larger than the channel diameter. Significantly, rearrangement of the ordered droplets therefore allows the synchronizing architecture to compensate for variations in droplet size. The presented synchronization architecture was optimized for a set range of droplet diameters. However, it is noted that larger droplets were more challenging to synchronize using the current architecture due to droplet splitting at the pillar array during packing. Nevertheless, modifications to the pillar array dimensions have been shown to be successful in synchronizing droplets with diameters up to $150 \mu \mathrm{m}$ (Fig. 4).

Another key factor in ensuring reliable droplet synchronization is the ability to allow for droplet overflow, which compensates for potential variations in droplet generation frequency. It was determined that under optimal conditions the device synchronizes approximately $17 \%$ of incoming droplets, with $83 \%$ of droplets being discarded. However, preliminary results

\begin{tabular}{|c|c|c|c|c|c|c|}
\hline $107 \mu \mathrm{m}$ & 81.04 & & & & & \\
\hline $110 \mu \mathrm{m}$ & 83.29 & 86.13 & & & & \\
\hline $118 \mu \mathrm{m}$ & 88.34 & 88.41 & 93.49 & & & \\
\hline $126 \mu \mathrm{m}$ & 92.00 & 99.41 & 97.78 & 100.00 & & \\
\hline $137 \mu \mathrm{m}$ & 87.74 & 80.80 & 81.80 & 99.85 & 97.30 & \\
\hline \multirow[t]{2}{*}{$150 \mu \mathrm{m}$} & 83.92 & 75.14 & 71.76 & 95.74 & 95.41 & 89.26 \\
\hline & $107 \mu \mathrm{m}$ & $110 \mu \mathrm{m}$ & $118 \mu \mathrm{m}$ & $126 \mu \mathrm{m}$ & $137 \mu \mathrm{m}$ & $150 \mu \mathrm{m}$ \\
\hline
\end{tabular}

\begin{tabular}{|c|c|c|c|c|c|c|}
\hline $107 \mu \mathrm{m}$ & 25.97 & & & & & \\
\hline $110 \mu \mathrm{m}$ & 24.75 & 20.32 & & & & \\
\hline $118 \mu \mathrm{m}$ & 12.78 & 17.83 & 9.44 & & & \\
\hline $126 \mu \mathrm{m}$ & 9.55 & 0.88 & 0.30 & 0.01 & & \\
\hline $137 \mu \mathrm{m}$ & 8.75 & 8.53 & 16.09 & 0.26 & 3.55 & \\
\hline \multirow[t]{2}{*}{$150 \mu \mathrm{m}$} & 5.66 & 6.58 & 10.56 & 6.46 & 6.27 & 12.06 \\
\hline & $107 \mu \mathrm{m}$ & $110 \mu \mathrm{m}$ & $118 \mu \mathrm{m}$ & $126 \mu \mathrm{m}$ & $137 \mu \mathrm{m}$ & $150 \mu \mathrm{m}$ \\
\hline
\end{tabular}

Fig. 3 (a) Dependence of synchronization performance (percentage of correctly synchronized droplets) on droplet diameter. Each tabulated value represents the mean synchronization performance from three independent experiments. Darker shading corresponds to increased synchronization performance. The optimal synchronization was achieved when two droplet types of equal size (126 $\mu \mathrm{m}$ diameter) were synchronized. This corresponds to a droplet diameter of around $58 \%$ of the total channel width. The reported value of $100.00 \%$ synchronization performance is rounded to two significant digits from a measured value of $99.997 \%$. (b) Standard deviation of the measured synchronization performance. Darker shading corresponds to a lower standard deviation. 


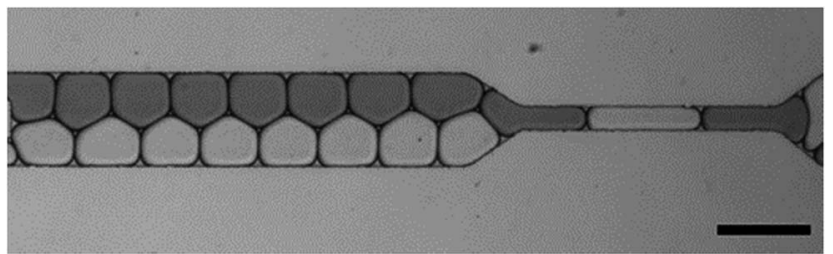

Fig. 4 Synchronization of droplets with diameters of up to $150 \mu \mathrm{m}$ (scale bar $-300 \mu \mathrm{m}$ ). Application of vacuum to the output allows for more accurate and faster synchronization. The device shown is a directly scaled up version of the previously described design.

suggest that the percentage of synchronized droplets can be significantly enhanced by altering the geometry in both the overflow and packing area (results not shown). Finally, it must be noted that overflow droplets are not discarded and can simply be collected, reintroduced and synchronized at a later time.

The balance of back-pressures between the two outlets has so far proven essential in achieving high accuracy synchronization. For example, an increase in back-pressure on "Out 1" due to an additional droplet unit operation (such as merging or incubation) must be compensated either by applying negative pressure on "Out 1" or increasing the back-pressure on "Out 2". Analogously the back-pressure within the two channels leading to "Out 2" should be kept identical. Accordingly, separation of these two channels to independent outlets allows for independent control of back-pressure, which will improve the synchronization performance, especially when operating with a high disparity in the droplet formation frequencies.

Long-term stability of the synchronization architecture was studied under the identified optimal conditions (with both droplets having a diameter of $126 \mu \mathrm{m}$ ). Specifically, over a period of 45 minutes, 47871 alternating droplets were produced with an error rate of less than $0.02 \%$. Synchronization of equal sized droplets entering the synchronization chamber at different flow rates was additionally evaluated to investigate the possibility of compensating for errors in droplet formation frequency. Synchronization was further characterized for conditions where the input droplet flow rates are markedly different. Fig. 5a shows that efficient synchronization is highly dependent on the maintenance of equal flow rates. As soon as the ratio of total flow rates between the two flow-focussing junctions deviates from unity there is a sharp decline in synchronization performance. Fig. $5 \mathrm{~b}$ shows the variation of the droplet synchronization frequency with the total flow rate through the synchronizer. As expected the number of synchronized droplets increases with higher flow-rates. In the current study we were able to achieve synchronization rates in excess of $31 \mathrm{~Hz}$. In general, it was found that under optimal conditions droplets could be synchronized at rates between $8.8 \mathrm{~Hz}$ and $31 \mathrm{~Hz}$ whilst maintaining a synchronization error below $1 \%$. It was also found that synchronization rates could be increased if negative pressure was applied to the "Out 1" channel, although this unsurprisingly required the modification of the flow conditions (data not shown). It is likely that higher synchronization rates would be achievable using higher flow rates than those investigated, although at the expense of increased error rates. In general, the dense packing of synchronized droplets required for an efficient synchronization operation depends on several factors including device geometry (i.e. the spacing and size of the pillars in the packing section), the back-pressure balance ("Out 1" vs. "Out 2") and the volumetric flow rate ratios during droplet formation. Spacing between densely packed, synchronized droplets could further be increased using an additional spacer oil inlet after synchronization, which resulted in a stream of $\mathrm{AB}$ droplets exhibiting uniform distances.

Although all results presented in this study were obtained using a surfactant concentration of $4 \%$, concentrations as low as $1 \%$ (data not shown) are sufficient to prevent spontaneous droplet fusion within the device. This reduction further facilitates down-stream merging of droplets, since most passive fusion methods require low surfactant concentrations. Nevertheless, many passive merging strategies do not provide the sufficiently low error rates to be useful in combination with the presented method. ${ }^{23}$ Accordingly, we suggest electrowetting as an ideal method for initiating droplet fusion through the continuous operation of electrode potentials.

Finally, streams of synchronized droplets could further be resplit into the constituent droplet streams (Fig. 6), a process previously reported by Surenjav and co-workers. ${ }^{57}$ This operation allows the production of two streams containing an equal number of droplets, thereby discretizing the number of droplets in a channel. Synchronized droplets can be kept in an alternate configuration in longer channels if a vacuum is applied to the elongated output 1 channel, lowering the back-pressure and thereby resulting in more stable droplet synchronization.

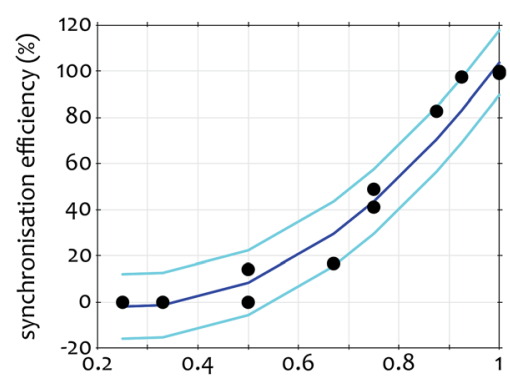

droplet 1 flow rate / droplet 2 flow rate

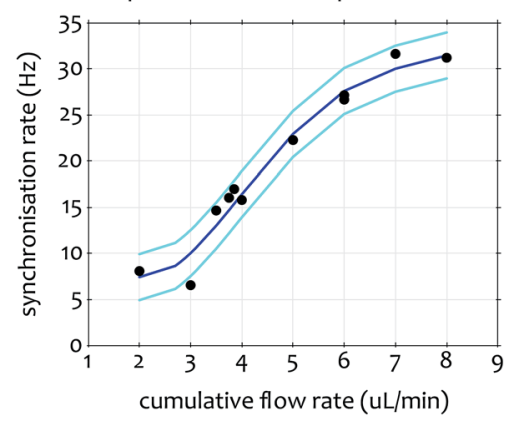

Fig. 5 (a) Dependence of synchronization performance on droplet size difference. A synchronization efficiency above $95 \%$ is only achieved if the two synchronized droplet types enter the chamber at equal flow rates. (b) Dependence of the synchronization rate on total flow rate, showing that throughput increases as a function of flow rate. 


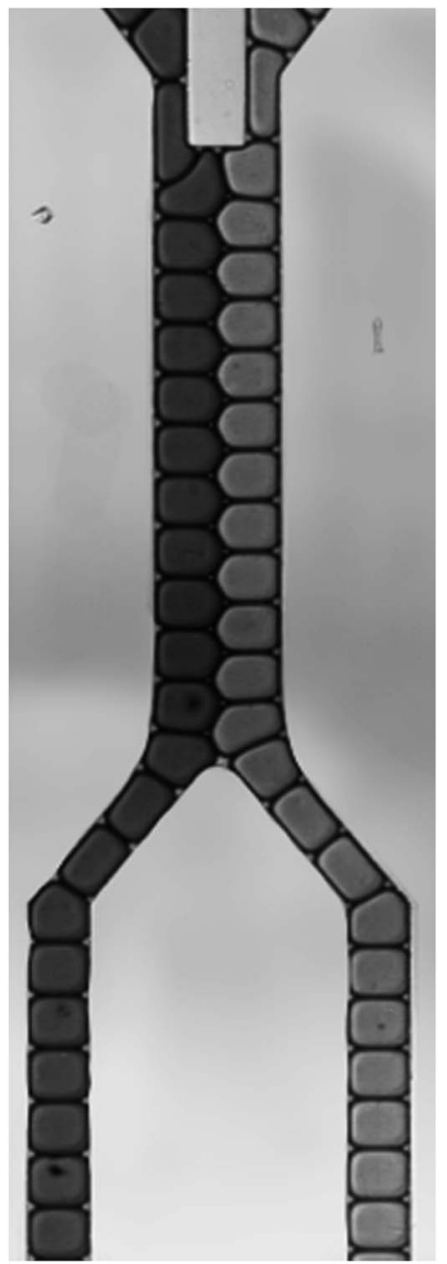

Fig. 6 Splitting a stream of synchronized droplets in an A-B alternate configuration into its constituent droplets and recombination of the separated streams. Direction of flow is from top to bottom (indicated by the arrow). This produces a situation where a uniform of droplets are flowing through the individual channel, but where each side contains droplets of different composition.

We believe the presented structure constitutes an interesting and high-efficiency tool for synchronizing droplets because of its inherently low error rate and ability to synchronize preformed droplets. We have demonstrated that the presented architecture is able to compensate for variations between the two synchronized streams in both droplet size and frequency. This architecture is therefore exceptionally useful for droplet merging and timing restoration, which are key operations in many biological and chemical assays.

\section{Conclusions}

We have demonstrated a passive microfluidic architecture to enable high efficiency droplet synchronization using preformed droplets. Using this architecture we are able to synchronize in excess of 45000 droplets in 45 minutes with an error rate below $0.02 \%$. We have further evaluated the synchronization performance of the device when synchronizing droplets of variable size and have established that excellent synchronization (with efficiencies in excess of $90 \%$ ) can be achieved over a broad droplet size range. We have also shown that droplet synchronization rates of up to $33 \mathrm{~Hz}$ are possible whilst maintaining a synchronization performance in excess of $99 \%$.

\section{Acknowledgements}

The authors would like to acknowledge ETH Zürich and the National Research Foundation of Korea (Global Research Laboratory Programme Grant K20904000004-10A0500-00410) for partial support. The authors declare no competing financial interest.

\section{References}

1 S. Y. Teh, R. Lin, L. H. Hung and A. P. Lee, Lab Chip, 2008, 8, 198-220.

2 M. T. Guo, A. Rotem, J. A. Heyman and D. A. Weitz, Lab Chip, 2012, 12, 2146-2155.

3 H. B. Yin and D. Marshall, Curr. Opin. Biotechnol., 2012, 23, 110-119.

4 S. Cho, D. K. Kang, J. Choo, A. J. deMello and S. I. Chang, BMB Rep., 2011, 44, 705-712.

5 A. B. Theberge, F. Courtois, Y. Schaerli, M. Fischlechner, C. Abell, F. Hollfelder and W. T. S. Huck, Angew. Chem., Int. Ed., 2010, 49, 5846-5868.

6 D. C. Duffy, J. C. McDonald, O. J. A. Schueller and G. M. Whitesides, Anal. Chem., 1998, 70, 4974-4984.

7 X. C. I. Solvas and A. deMello, Chem. Commun., 2011, 47, 1936-1942.

8 T. Thorsen, R. W. Roberts, F. H. Arnold and S. R. Quake, Phys. Rev. Lett., 2001, 86, 4163-4166.

9 Y. J. Wang, W. Y. Lin, K. Liu, R. J. Lin, M. Selke, H. C. Kolb, N. G. Zhang, X. Z. Zhao, M. E. Phelps, C. K. F. Shen, K. F. Faull and H. R. Tseng, Lab Chip, 2009, 9, 2281-2285.

10 M. R. Bringer, C. J. Gerdts, H. Song, J. D. Tice and R. F. Ismagilov, Philos. Trans. R. Soc., A, 2004, 362, 10871104.

11 P. Garstecki, I. Gitlin, W. DiLuzio, G. M. Whitesides, E. Kumacheva and H. A. Stone, Appl. Phys. Lett., 2004, 85, 2649-2651.

12 M. Hashimoto, P. Garstecki, H. A. Stone and G. M. Whitesides, Soft Matter, 2008, 4, 1403-1413.

13 Y. Ding, X. Casadevall i Solvas and A. deMello, Analyst, 2015, 140, 414-421.

14 D. R. Link, S. L. Anna, D. A. Weitz and H. A. Stone, Phys. Rev. Lett., 2004, 92, 054503.

15 Y. C. Tan, J. S. Fisher, A. I. Lee, V. Cristini and A. P. Lee, $L a b$ Chip, 2004, 4, 292-298.

16 R. C. R. Wootton and A. J. Demello, Nature, 2012, 483, 43-44. 17 B. Kintses, L. D. van Vliet, S. R. A. Devenish and F. Hollfelder, Curr. Opin. Chem. Biol., 2010, 14, 548-555.

18 D. N. Adamson, D. Mustafi, J. X. J. Zhang, B. Zheng and R. F. Ismagilov, Lab Chip, 2006, 6, 1178-1186. 
19 G. F. Christopher, J. Bergstein, N. B. End, M. Poon, C. Nguyen and S. L. Anna, Lab Chip, 2009, 9, 1102-1109.

20 P. M. Korczyk, L. Derzsi, S. Jakiela and P. Garstecki, Lab Chip, 2013, 13, 4096-4102.

21 A. R. Abate, T. Hung, P. Mary, J. J. Agresti and D. A. Weitz, Proc. Natl. Acad. Sci. U. S. A., 2010, 107, 19163-19166.

22 C. Holtze, A. C. Rowat, J. J. Agresti, J. B. Hutchison, F. E. Angile, C. H. J. Schmitz, S. Koster, H. Duan, K. J. Humphry, R. A. Scanga, J. S. Johnson, D. Pisignano and D. A. Weitz, Lab Chip, 2008, 8, 1632-1639.

23 L. Mazutis, J. C. Baret and A. D. Griffiths, Lab Chip, 2009, 9, 2665-2672.

24 V. Trivedi, A. Doshi, G. K. Kurup, E. Ereifej, P. J. Vandevord and A. S. Basu, Lab Chip, 2010, 10, 2433-2442.

25 J. Clausell-Tormos, D. Lieber, J. C. Baret, A. El-Harrak, O. J. Miller, L. Frenz, J. Blouwolff, K. J. Humphry, S. Koster, H. Duan, C. Holtze, D. A. Weitz, A. D. Griffiths and C. A. Merten, Chem. Biol., 2008, 15, 875.

26 L. Frenz, K. Blank, E. Brouzes and A. D. Griffiths, Lab Chip, 2009, 9, 1344-1348.

27 J. C. Baret, O. J. Miller, V. Taly, M. Ryckelynck, A. El-Harrak, L. Frenz, C. Rick, M. L. Samuels, J. B. Hutchison, J. J. Agresti, D. R. Link, D. A. Weitz and A. D. Griffiths, Lab Chip, 2009, 9, 1850-1858.

28 C. Lee, J. Lee, H. H. Kim, S. Y. Teh, A. Lee, I. Y. Chung, J. Y. Park and K. K. Shung, Lab Chip, 2012, 12, 2736-2742.

29 Z. N. Cao, F. Y. Chen, N. Bao, H. C. He, P. S. Xu, S. Jana, S. H. Jung, H. Z. Lian and C. Lu, Lab Chip, 2013, 13, 171-178. 30 X. L. Mao and T. J. Huang, Lab Chip, 2012, 12, 4006-4009.

31 X. Niu, S. Gulati, J. B. Edel and A. J. deMello, Lab Chip, 2008, 8, 1837-1841.

32 M. Zagnoni, C. N. Baroud and J. M. Cooper, Phys. Rev. E: Stat., Nonlinear, Soft Matter Phys., 2009, 80, 046303.

33 E. Um and J. K. Park, Lab Chip, 2009, 9, 207-212.

34 L. M. Fidalgo, C. Abell and W. T. S. Huck, Lab Chip, 2007, 7, 984-986.

35 T. Nisisako, S. A. Portonovo and J. J. Schmidt, Analyst, 2013, 138, 6793-6800.

36 M. Zagnoni and J. M. Cooper, Lab Chip, 2010, 10, 3069-3073.

37 R. M. Schoeman, E. W. M. Kemna, F. Wolbers and A. van den Berg, Electrophoresis, 2014, 35, 385-392.

38 T. Rob and D. J. Wilson, J. Am. Soc. Mass Spectrom., 2009, 20, 124-130.
39 Z. Michalak, D. Fartash, N. Haque and S. Lee, CrystEngComm, 2012, 14, 7865-7868.

40 H. Zec, T. D. Rane and T. H. Wang, Lab Chip, 2012, 12, 30553062.

41 A. C. Hatch, J. S. Fisher, A. R. Tovar, A. T. Hsieh, R. Lin, S. L. Pentoney, D. L. Yang and A. P. Lee, Lab Chip, 2011, 11, 3838-3845.

42 A. Bransky, N. Korin, M. Khoury and S. Levenberg, Lab Chip, 2009, 9, 516-520.

43 K. Churski, T. S. Kaminski, S. Jakiela, W. Kamysz, W. Baranska-Rybak, D. B. Weibel and P. Garstecki, Lab Chip, 2012, 12, 1629-1637.

44 K. Ahn, J. Agresti, H. Chong, M. Marquez and D. A. Weitz, Appl. Phys. Lett., 2006, 88, 264105.

45 J. Hong, M. Choi, J. B. Edel and A. J. deMello, Lab Chip, 2010, 10, 2702-2709.

46 L. F. Xu, H. Lee, R. Panchapakesan and K. W. Oh, Lab Chip, 2012, 12, 3936-3942.

47 B. Ahn, K. Lee, H. Lee, R. Panchapakesan, L. F. Xu, J. Xu and K. W. Oh, Lab Chip, 2011, 11, 3915-3918.

48 B. Zheng, J. D. Tice and R. F. Ismagilov, Anal. Chem., 2004, 76, 4977-4982.

49 L. H. Hung, K. M. Choi, W. Y. Tseng, Y. C. Tan, K. J. Shea and A. P. Lee, Lab Chip, 2006, 6, 174-178.

50 E. Um, M. E. Rogers and H. A. Stone, Lab Chip, 2013, 13, 4674-4680.

51 L. Frenz, J. Blouwolff, A. D. Griffiths and J. C. Baret, Langmuir, 2008, 24, 12073-12076.

52 V. Chokkalingam, S. Herminghaus and R. Seemann, Appl. Phys. Lett., 2008, 93, 254101.

53 R. Dangla, E. Fradet, Y. Lopez and C. N. Baroud, J. Phys. D: Appl. Phys., 2013, 46, 114003.

54 R. M. Lorenz, G. S. Fiorini, G. D. M. Jeffries, D. S. W. Lim, M. Y. He and D. T. Chiu, Anal. Chim. Acta, 2008, 630, 124130.

55 M. Prakash and N. Gershenfeld, Science, 2007, 315, 832-835.

56 C. Priest, S. Herminghaus and R. Seemann, Appl. Phys. Lett., 2006, 88, 024106.

57 E. Surenjav, S. Herminghaus, C. Priest and R. Seemann, Appl. Phys. Lett., 2009, 95, 154104.

58 V. Labrot, M. Schindler, P. Guillot, A. Colin and M. Joanicot, Biomicrofluidics, 2009, 3, 012804. 